PHYSICAL REVIEW D 89, 055027 (2014)

\title{
GUT predictions for quark-lepton Yukawa coupling ratios with messenger masses from non-singlets
}

\author{
Stefan Antusch, ${ }^{1,2^{*}}$ Stephen F. King, ${ }^{3 \dagger}$ and Martin Spinrath ${ }^{4,5 \sharp}$ \\ ${ }^{1}$ Department of Physics, University of Basel, Klingelbergstrasse 82, CH-4056 Basel, Switzerland \\ ${ }^{2}$ Max-Planck-Institut für Physik (Werner-Heisenberg-Institut), \\ Föhringer Ring 6, D-80805 München, Germany \\ ${ }^{3}$ School of Physics and Astronomy, University of Southampton, SO17 1BJ Southampton, United Kingdom \\ ${ }^{4}$ SISSA/ISAS and INFN, Via Bonomea 265, I-34136 Trieste, Italy \\ ${ }^{5}$ Institut für Theoretische Teilchenphysik, Karlsruhe Institute of Technology, \\ Engesserstraße 7, D-76131 Karlsruhe, Germany
}

(Received 27 November 2013; published 27 March 2014)

\begin{abstract}
We propose new predictions from grand unified theories (GUTs) [applicable to both supersymmetric (SUSY) and non-SUSY models] for the ratios of quark and lepton Yukawa couplings. These new predictions arise from splitting the masses of the messenger fields for the GUT-scale Yukawa operators by Clebsch-Gordan factors from GUT symmetry breaking. This has the effect that these factors enter inversely in the predicted quark-lepton Yukawa coupling ratios, leading to new possible GUT predictions. We systematically construct the new predictions that can be realized in this way in SU(5) GUTs and Pati-Salam unified theories and discuss model building applications.
\end{abstract}

DOI: $10.1103 /$ PhysRevD.89.055027

PACS numbers: 12.10.-g, 12.15.Ff, 12.10.Dm

\section{INTRODUCTION}

In unified theories of fermion masses and mixings, such as in SU(5) grand unified theories (GUTs) [1] or Pati-Salam (PS) models [2], the fermions of the Standard Model (SM) (plus in the latter case right-handed neutrinos) are unified in joint representations of the enlarged unified gauge symmetry group. When this symmetry gets broken to the SM, this can result in predictions for the ratios of the entries of the quark and lepton Yukawa matrices at the unification scale $M_{\mathrm{GUT}}$. Typical examples for such predictions are bottom-tau Yukawa coupling unification [1], i.e. $y_{b}=y_{\tau}$, at $M_{\mathrm{GUT}}$, or the Georgi-Jarlskog relation $y_{\mu} / y_{s}=3$ [3]. Both ratios can emerge from renormalizable GUT operators for Yukawa couplings in the context of SU(5) GUTs or PatiSalam models [seen as a step towards SO(10) GUTs]. The phenomenology of these relations was studied quite extensively; for some recent references, see, for instance, [4-8].

However, in models for fermion masses and mixings which aim at explaining the hierarchies between the quark and charged lepton Yukawa couplings of the different generations, the Yukawa couplings smaller than $\mathcal{O}(1)$ are preferably generated by effective operators, realized by the exchange of heavy messenger fields in GUT representations. This applies to the Yukawa couplings of the first and second generations but also to the bottom and tau Yukawa couplings in supersymmetric (SUSY) models with small or moderate $\tan \beta$ or in non-SUSY models. In the case of

\footnotetext{
*stefan.antusch@unibas.ch

king@soton.ac.uk

\#martin.spinrath@kit.edu
}

SUSY models, we assume that the low energy theory corresponds to the minimal supersymmetric Standard Model (MSSM).

The new possibilities which arise from such operators have been discussed in the context of SU(5) GUTs in [9] and in Pati-Salam models in [9-11] with dimension 6 and dimension 5 operators, respectively. For similar studies in $\mathrm{SO}(10)$ see, for instance, [12] or more recently [13]. It has been shown in [9] that in many SUSY scenarios the new SU(5) relations like $y_{\tau} / y_{b}=-\frac{3}{2}$ or $y_{\mu} / y_{s}=\frac{9}{2}$ or 6 are often favored compared to bottom-tau Yukawa unification or the Georgi-Jarlskog relation. The implications of these new relations were studied, for instance, in [14-16]. In any case, towards making progress in building SUSY or non-SUSY GUT models of flavor, it is important to be aware of the full set of possibilities and to study their phenomenological consequences.

In this paper, we discuss a new way of obtaining predictions for the GUT-scale ratios of quark and lepton Yukawa couplings. These new possibilities arise from splitting the masses of the messenger fields for the GUT-scale Yukawa operators by Clebsch-Gordan (CG) factors from GUT symmetry breaking. This has the effect that these factors enter inversely in the predicted quarklepton mass relations, leading to new possible predictions. We systematically list the new predictions that can be realized in this way in SU(5) GUTs and Pati-Salam unified theories and discuss model building applications.

\section{PREDICTIONS FROM SU(5) UNIFICATION}

In [9] some of the authors considered SU(5) GUT mass ratios from dimension 5 operators. The approach can be 


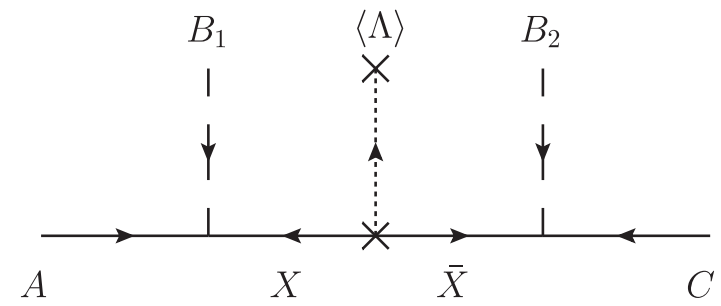

FIG. 1. Diagrammatic representation of the operators giving Yukawa coupling ratios at the GUT scale. $A$ and $C$ are matter fields while $B_{1}$ and $B_{2}$ are Higgs fields. $\langle\Lambda\rangle$ represents the messenger mass term. If $\Lambda$ is a total singlet, one could directly write down a mass like in [9]. Otherwise $\Lambda$ should transform as an adjoint of the GUT symmetry splitting the masses of the components of the messenger fields.

most easily explained looking at Fig. 1. In the previous publication the vacuum expectation value (VEV) of $\Lambda$ was not considered or in other words the mass of the messenger fields pair $X$ and $\bar{X}$ was assumed to transform trivially as a gauge singlet. The external fields $A, B_{1}, B_{2}$ and $C$ (note that we use here a slightly different notation) were assigned to various GUT representations. In particular, one was the five-dimensional matter representation of $\mathrm{SU}(5)$ :

$$
F=\overline{\mathbf{5}}=\left(d_{R}^{c} d_{B}^{c} d_{G}^{c} e-\nu\right)_{L},
$$

one was the ten-dimensional matter representation of $\mathrm{SU}(5)$ :

$$
T=\mathbf{1 0}=\frac{1}{\sqrt{2}}\left(\begin{array}{ccccc}
0 & -u_{G}^{c} & u_{B}^{c} & -u_{R} & -d_{R} \\
u_{G}^{c} & 0 & -u_{R}^{c} & -u_{B} & -d_{B} \\
-u_{B}^{c} & u_{R}^{c} & 0 & -u_{G} & -d_{G} \\
u_{R} & u_{B} & u_{G} & 0 & -e^{c} \\
d_{R} & d_{B} & d_{G} & e^{c} & 0
\end{array}\right)_{L}
$$

and one was the $\mathrm{SU}(5)$ representation containing the Higgs doublet. This can sit either in a five- or in a 45-dimensional representation

$$
\begin{aligned}
\left(\bar{h}_{5}\right)^{a} & =\overline{\mathbf{5}}^{a}, \quad\left\langle\left(\bar{h}_{5}\right)^{5}\right\rangle=v_{5}, \\
\left(\bar{h}_{45}\right)_{c}^{a b} & =-\left(\bar{h}_{45}\right)_{c}^{b a}=\overline{\mathbf{4 5}}_{c}^{a b}, \\
\left\langle\left(\bar{h}_{45}\right)_{j}^{i 5}\right\rangle & =v_{45}\left(\delta_{j}^{i}-4 \delta^{i 4} \delta_{j 4}\right),
\end{aligned}
$$

where $a, b=1, \ldots, 5, \quad \alpha=1,2, \quad 3, \quad \beta=4, \quad 5$ and $i, j=1, \ldots, 4$. The fourth external field of the diagram in Fig. 1 was assigned to a GUT symmetry breaking Higgs field, like the adjoint

$$
\begin{aligned}
\left(H_{24}\right)_{b}^{a} & =\mathbf{2 4}_{b}^{a}, \\
\left\langle\left(H_{24}\right)_{b}^{a}\right\rangle & =V_{24}\left(2 \delta_{\alpha}^{a} \delta_{b}^{\alpha}-3 \delta_{\beta}^{a} \delta_{b}^{\beta}\right),
\end{aligned}
$$

where again $a, b=1, \ldots, 5, \alpha=1,2,3$ and $\beta=4,5$.
If we identify $A$ and $C$ with the matter representations $F$ and $T$, then there are new possible Yukawa coupling relations beyond the renormalizable ones. With the VEV of the additional GUT Higgs field $H_{24}$ pointing in the hypercharge direction we found, for instance, the new GUT predictions $\left(Y_{e}\right)_{i j} /\left(Y_{d}\right)_{i j}=-3 / 2,9 / 2$ and 6 , depending on which representation the $H_{24}$ field couples to and on which representation contains the SM Higgs doublet. In the SUSY case the fields $A$ and $C$ would be fermionic components and the fields $B_{1}, B_{2}$ and $\Lambda$ scalar components of the respective superfields. As already noted, our results also apply to the non-SUSY case.

In this work we want to extend the above approach to include the case that the mass of the messenger pair $X$ and $\bar{X}$ is not generated by a gauge singlet $\Lambda_{1}$ but by a GUT nonsinglet, e.g. by a field in the adjoint representation of $S U(5)$, $\Lambda_{24}$. In this particular case the masses of the components of the messenger fields are proportional to their respective hypercharges. More generally, when the heavy messenger fields (which in general have masses split by CG factors according to our approach) are integrated out, these CG factors will enter inversely in the considered down-type quark and charged lepton Yukawa matrix elements, which can lead to new GUT predictions for the ratios $\left(Y_{e}\right)_{i j} /\left(Y_{d}\right)_{i j}$ beyond the ones considered in [9]. Note that in the diagram of Fig. 1 the field $\Lambda$, which acquires a VEV, should not be viewed as an external field of an effective operator. When $\Lambda$ acquires its VEV, it generates the mass term for the messenger pair $X$ and $\bar{X}$ and only then the messengers can be integrated out to obtain an effective operator. Consequently, the VEV $\langle\Lambda\rangle$ will appear inversely in the effective operator.

In general, when $\Lambda$ is not a singlet but e.g. in the adjoint representation, the components of the messenger pair $X$ and $\bar{X}$ receive masses (around the GUT scale in size), which are split by CG coefficients. Below this scale, since the GUT symmetry is now already spontaneously broken, also the fields in the matter and Higgs representations are split into their SM representations and, furthermore, the Higgs fields $B_{1}$ and $B_{2}$ acquire their VEVs which are smaller than the VEV of $\Lambda$. One of the fields $B_{1}$ and $B_{2}$ contains the SM Higgs field and the other one may either be a GUT singlet or in a 24- or 75-dimensional representation. If the field is not a GUT singlet, it introduces another CG factor. In total, we obtain a prediction for the ratio $\left(Y_{e}\right)_{i j} /\left(Y_{d}\right)_{i j}$ of the considered charged lepton and down-type quark Yukawa matrix element.

For illustration, let us study an instructive example: suppose $A=F, B_{1}=H_{1}, B_{2}=\bar{h}_{5}$ and $C=T . H_{1}$ is a GUT singlet acquiring a heavy VEV satisfying $\left\langle H_{1}\right\rangle<M_{\text {GUT }}$. In a flavor model this could be for example a flavon. If the messenger masses have a trivial mass proportional to $\left\langle\Lambda_{1}\right\rangle$, we get the ordinary bottom-tau Yukawa coupling unification. But we assume now that the symmetries are such that the pair $X$ and $\bar{X}$, which are 
TABLE I. Summary of possible SU(5) predictions for the GUTscale Yukawa coupling ratios $\left(Y_{e}\right)_{i j} /\left(Y_{d}\right)_{i j}$. The new relations compared to [9] are shown in bold. For more details about which operator gives which ratio, see Table II.

\begin{tabular}{lc}
\hline \hline Operator dimension & $\left(Y_{e}\right)_{i j} /\left(Y_{d}\right)_{i j}$ \\
\hline 4 & 1 \\
5 & -3 \\
& $\mathbf{1 / 6}$ \\
& $-1 / 2$ \\
& $-\mathbf{2} / \mathbf{3}$ \\
1 \\
& $\pm 3 / 2$ \\
& -3 \\
$9 / 2$ \\
6 \\
\end{tabular}

five-dimensional representations of $\mathrm{SU}(5)$, get their mass from $\left\langle\Lambda_{24}\right\rangle$. To be more precise, when the down-type quarklike components of $X$ and $\bar{X}$ obtain a mass $-2 M$, then the leptonic components have the mass $3 M$ (with $M$ around $\left.M_{\mathrm{GUT}}\right)$. At the GUT scale we therefore get in the Lagrangian the effective operators

$$
\mathcal{L} \supset Y_{i j} \frac{\left\langle H_{1}\right\rangle}{M}\left(\frac{1}{3} L_{i} \bar{e}_{j}^{c}-\frac{1}{2} Q_{j} \bar{d}_{i}^{c}\right) h_{d}+\text { H.c. },
$$

which we have written down in terms of Standard Model fields and where $i, j$ are family indices. Taking the ratio now yields $\left(Y_{e}\right)_{i j} /\left(Y_{d}\right)_{i j}=-2 / 3$, which is a new result not contained in [9].

In Table I we have collected all the ratios we have found using this approach where we have restricted $\Lambda$ to be not larger than the adjoint and $X$ not to be larger than the $\mathbf{5 0}$ of SU(5). The relations which are new compared to the previous publication are highlighted in bold. The involved fields can be read off from Table II where we give the explicit representations for $A, B_{1}, B_{2}, C, X$ and $\Lambda$ from Fig. 1 and their prediction for $\left(Y_{e}\right)_{i j} /\left(Y_{d}\right)_{i j}$.

There are a few more comments in order. First of all, in Table II we give only the cases where the messenger fields connect the pairs $\left(A, B_{1}\right)$ and $\left(C, B_{2}\right)$ because only in this case the messengers are matterlike and we get new relations. The other case is given in the Appendix; see especially Fig. 4. In that case the messengers act as effective Higgs fields which obtain an induced VEV and we only get the well-known dimension 4 results for the Yukawa couplings.

Then we also want to mention the case where $X$ and $\bar{X}$ are not in conjugated representations to each other. For example $X=\mathbf{5}$ and $\bar{X}=\overline{\mathbf{4 5}}$ is possible if $\Lambda$ is the adjoint of SU(5). In this case 40 of the 45 components of $\bar{X}$ remain massless which should not be the case to avoid light exotics. In principle one might add a $X^{\prime}=\mathbf{4 5}$ field to overcome this problem but this might introduce a different Yukawa coupling ratio and hence spoil the clean prediction
TABLE II. Resulting predictions for the SU(5) GUT-scale Yukawa coupling ratios $\left(Y_{e}\right)_{i j} /\left(Y_{d}\right)_{i j}$ from the diagram in Fig. 1; for more details see main text.

\begin{tabular}{|c|c|c|c|c|}
\hline$\left(A, B_{1}\right)$ & $\left(C, B_{2}\right)$ & $X$ & $\Lambda$ & $\left(Y_{e}\right)_{i j} /\left(Y_{d}\right)_{i j}$ \\
\hline$\left(F, \bar{h}_{5}\right)$ & $\left(T, H_{1}\right)$ & 10 & $\Lambda_{1}$ & 1 \\
\hline$\left(F, \bar{h}_{45}\right)$ & $\left(T, H_{1}\right)$ & 10 & $\Lambda_{1}$ & -3 \\
\hline$\left(F, \bar{h}_{5}\right)$ & $\left(T, H_{24}\right)$ & 10 & $\Lambda_{1}$ & 6 \\
\hline$\left(F, \bar{h}_{5}\right)$ & $\left(T, H_{24}\right)$ & 15 & $\Lambda_{1}$ & 0 \\
\hline$\left(F, \bar{h}_{5}\right)$ & $\left(T, H_{75}\right)$ & 10 & $\Lambda_{1}$ & -3 \\
\hline$\left(F, \bar{h}_{45}\right)$ & $\left(T, H_{24}\right)$ & 10 & $\Lambda_{1}$ & -18 \\
\hline$\left(F, \bar{h}_{45}\right)$ & $\left(T, H_{24}\right)$ & 40 & $\Lambda_{1}$ & 0 \\
\hline$\left(F, \bar{h}_{45}\right)$ & $\left(T, H_{75}\right)$ & 10 & $\Lambda_{1}$ & 9 \\
\hline$\left(F, \bar{h}_{45}\right)$ & $\left(T, H_{75}\right)$ & 40 & $\Lambda_{1}$ & 0 \\
\hline$\left(F, H_{1}\right)$ & $\left(T, \bar{h}_{5}\right)$ & 5 & $\Lambda_{1}$ & 1 \\
\hline$\left(F, H_{1}\right)$ & $\left(T, \bar{h}_{45}\right)$ & 5 & $\Lambda_{1}$ & -3 \\
\hline$\left(F, H_{24}\right)$ & $\left(T, \bar{h}_{5}\right)$ & 5 & $\Lambda_{1}$ & $-3 / 2$ \\
\hline$\left(F, H_{24}\right)$ & $\left(T, \bar{h}_{5}\right)$ & 45 & $\Lambda_{1}$ & $3 / 2$ \\
\hline$\left(F, H_{75}\right)$ & $\left(T, \bar{h}_{5}\right)$ & 45 & $\Lambda_{1}$ & -3 \\
\hline$\left(F, H_{24}\right)$ & $\left(T, \bar{h}_{45}\right)$ & 5 & $\Lambda_{1}$ & $9 / 2$ \\
\hline$\left(F, H_{24}\right)$ & $\left(T, \bar{h}_{45}\right)$ & 45 & $\Lambda_{1}$ & $-1 / 2$ \\
\hline$\left(F, H_{75}\right)$ & $\left(T, \bar{h}_{45}\right)$ & 45 & $\Lambda_{1}$ & 1 \\
\hline$\left(F, H_{75}\right)$ & $\left(T, \bar{h}_{45}\right)$ & 50 & $\Lambda_{1}$ & 0 \\
\hline$\left(F, \bar{h}_{5}\right)$ & $\left(T, H_{1}\right)$ & 10 & $\Lambda_{24}$ & $1 / 6$ \\
\hline$\left(F, \bar{h}_{45}\right)$ & $\left(T, H_{1}\right)$ & 10 & $\Lambda_{24}$ & $-1 / 2$ \\
\hline$\left(F, \bar{h}_{5}\right)$ & $\left(T, H_{24}\right)$ & 10 & $\Lambda_{24}$ & 1 \\
\hline$\left(F, \bar{h}_{5}\right)$ & $\left(T, H_{24}\right)$ & 15 & $\Lambda_{24}$ & 0 \\
\hline$\left(F, \bar{h}_{5}\right)$ & $\left(T, H_{75}\right)$ & 10 & $\Lambda_{24}$ & $-1 / 2$ \\
\hline$\left(F, \bar{h}_{45}\right)$ & $\left(T, H_{24}\right)$ & 10 & $\Lambda_{24}$ & -3 \\
\hline$\left(F, \bar{h}_{45}\right)$ & $\left(T, H_{24}\right)$ & 40 & $\Lambda_{24}$ & 0 \\
\hline$\left(F, \bar{h}_{45}\right)$ & $\left(T, H_{75}\right)$ & 10 & $\Lambda_{24}$ & $3 / 2$ \\
\hline$\left(F, \bar{h}_{45}\right)$ & $\left(T, H_{75}\right)$ & 40 & $\Lambda_{24}$ & 0 \\
\hline$\left(F, H_{1}\right)$ & $\left(T, \bar{h}_{5}\right)$ & 5 & $\Lambda_{24}$ & $-2 / 3$ \\
\hline$\left(F, H_{1}\right)$ & $\left(T, \bar{h}_{45}\right)$ & 5 & $\Lambda_{24}$ & 2 \\
\hline$\left(F, H_{24}\right)$ & $\left(T, \bar{h}_{5}\right)$ & 5 & $\Lambda_{24}$ & 1 \\
\hline$\left(F, H_{24}\right)$ & $\left(T, \bar{h}_{45}\right)$ & 5 & $\Lambda_{24}$ & -3 \\
\hline$\left(F, H_{75}\right)$ & $\left(T, \bar{h}_{45}\right)$ & 50 & $\Lambda_{24}$ & 0 \\
\hline
\end{tabular}

for $\left(Y_{e}\right)_{i j} /\left(Y_{d}\right)_{i j}$ so that we do not discuss this case here any further.

Furthermore, it can happen that the product of $X$ with $\bar{X}$ contains more than one adjoint representation. To be more precise this happens in SU(5) if the messengers are in 40- or 45-dimensional representations and their products contain two different adjoint representations. Evaluating the effective operator then might yield two different ratios $\left(Y_{e}\right)_{i j} /\left(Y_{d}\right)_{i j}$ depending on how you contract the indices. For example, the combination $\left(A, B_{1}\right)=\left(F, H_{75}\right)$ with $\left(C, B_{2}\right)=\left(T, \bar{h}_{5}\right)$ and $X=\mathbf{4 5}$ yields the two different ratios $\left(Y_{e}\right)_{i j} /\left(Y_{d}\right)_{i j}=2$ and $\left(Y_{e}\right)_{i j} /\left(Y_{d}\right)_{i j}=12$. Hence, in these cases no proper prediction is possible and we discard them from our tables. Nevertheless, for the 40-dimensional messengers the ratio does not depend on the SU(5) index contraction so that the ratio remains unique and we list it in the tables. ${ }^{1}$

\footnotetext{
${ }^{1}$ We want to thank Vinzenz Maurer for bringing this point to our attention.
} 
At this point we would like to make another remark. There may of course be corrections to the predicted ratios, coming for example from further operators at a higher level in the operator expansion, from GUT threshold corrections or from the effect of possible large GUT representations on the gauge coupling evolution. The size of such corrections has to be estimated when an explicit model is constructed, as was done, for instance in an $\mathrm{SO}(10)$ model [17].

\section{PREDICTIONS FROM PATI-SALAM UNIFICATION}

For the case of PS models we followed in principle the same approach as for $\mathrm{SU}(5)$; see the previous section. Therefore we will not describe the approach here again in detail but instead just define all the fields and representations of the matter, Higgs and $\Lambda$ fields involved.

The PS group $\mathrm{SU}(4)_{C} \times \mathrm{SU}(2)_{L} \times \mathrm{SU}(2)_{R}$ is left-right symmetric and the matter fields of the Standard Model are contained in two representations

$$
\begin{aligned}
& R_{\alpha a}^{i}=(\mathbf{4}, \mathbf{2}, \mathbf{1})^{i}=\left(\begin{array}{cccc}
u_{L}^{R} & u_{L}^{B} & u_{L}^{G} & \nu_{L} \\
d_{L}^{R} & d_{L}^{B} & d_{L}^{G} & e_{L}^{-}
\end{array}\right)^{i}, \\
& \bar{R}^{i \alpha x}=(\overline{\mathbf{4}}, \mathbf{1}, \overline{\mathbf{2}})^{i}=\left(\begin{array}{llll}
\bar{d}_{R}^{R} & \bar{d}_{R}^{B} & \bar{d}_{R}^{G} & e_{R}^{+} \\
\bar{u}_{R}^{R} & \bar{u}_{R}^{B} & \bar{u}_{R}^{G} & \bar{\nu}_{R}
\end{array}\right)^{i},
\end{aligned}
$$

where $\alpha=1, \ldots, 4$ is an $\mathrm{SU}(4)_{C}$ index, $a, x=1,2$ are $\mathrm{SU}(2)_{L, R}$ indices and $i=1,2,3$ is a family index. The fields in $R^{i}$ form $\mathrm{SU}(2)_{L}$ doublets and the fields in $\bar{R}^{i}$ $S U(2)_{R}$ doublets as indicated by the indices $L$ and $R$.

The MSSM Higgs doublets are contained in the bidoublet representation

$$
\left(h_{1}\right)_{x}^{a}=(\mathbf{1}, \overline{\mathbf{2}}, \mathbf{2})=\left(\begin{array}{cc}
h_{u}^{+} & h_{d}^{0} \\
h_{u}^{0} & h_{d}^{-}
\end{array}\right),
$$

where the components $h_{u}^{0}$ and $h_{d}^{0}$ acquire the electroweak symmetry breaking VEVs. To get the Georgi-Jarlskog relation at the renormalizable level the Higgs doublets are contained in the $h_{15}=(\mathbf{1 5}, \overline{\mathbf{2}}, \mathbf{2})$ field where the VEV points into the direction of $B-L$ due to the tracelessness of the adjoint of $\mathrm{SU}(4)_{C}$.

The PS symmetry is broken by the two Higgs fields

$$
\begin{gathered}
H^{\alpha b}=(\mathbf{4}, \mathbf{1}, \mathbf{2})=\left(\begin{array}{llll}
u_{H}^{R} & u_{H}^{B} & u_{H}^{G} & \nu_{H} \\
d_{H}^{R} & d_{H}^{B} & d_{H}^{G} & e_{H}^{-}
\end{array}\right), \\
\bar{H}_{\alpha x}=(\overline{\mathbf{4}}, \mathbf{1}, \overline{\mathbf{2}})=\left(\begin{array}{llll}
\bar{d}_{H}^{R} & \bar{d}_{H}^{B} & \bar{d}_{H}^{G} & e_{H}^{+} \\
\bar{u}_{H}^{R} & \bar{u}_{H}^{B} & \bar{u}_{H}^{G} & \bar{\nu}_{H}
\end{array}\right),
\end{gathered}
$$

where the GUT symmetry breaking VEV points in the directions $\left\langle\nu_{H}\right\rangle$ and $\left\langle\bar{\nu}_{H}\right\rangle$. We have also considered the cases where adjoints of PS acquire GUT-scale VEVs

$$
\begin{array}{ll}
\phi_{1}^{+}=(\mathbf{1}, \mathbf{1}, \mathbf{1}), & \phi_{15}^{+}=(\mathbf{1 5}, \mathbf{1}, \mathbf{1}), \\
\phi_{1}^{-}=(\mathbf{1}, \mathbf{1}, \mathbf{3}), & \phi_{15}^{-}=(\mathbf{1 5}, \mathbf{1}, \mathbf{3}),
\end{array}
$$

which would not break PS to the Standard Model. However, in a more complete theory, one could regard them as effective combinations of $H \bar{H}$, as discussed in [11].

In the PS case we have more possibilities for the $\Lambda$ fields because we can take adjoints of $\mathrm{SU}(4)_{C}$ and $\mathrm{SU}(2)_{R}$ and combinations of them

$$
\begin{array}{ll}
\Lambda_{1}^{+}=(\mathbf{1}, \mathbf{1}, \mathbf{1}), & \Lambda_{15}^{+}=(\mathbf{1 5}, \mathbf{1}, \mathbf{1}), \\
\Lambda_{1}^{-}=(\mathbf{1}, \mathbf{1}, \mathbf{3}), & \Lambda_{15}^{-}=(\mathbf{1 5}, \mathbf{1}, \mathbf{3}) .
\end{array}
$$

The results for PS are summarized in Table III and the detailed operators are listed in Tables IV and V. Also for the PS case we find new relations compared to the previous study [9]; for example we find the ratio $\left(Y_{e}\right)_{i j} /\left(Y_{d}\right)_{i j}=$ $3 / 2$ which is a promising ratio for the third generation as it was noted in [9] but where (with a singlet $\Lambda$ ) this ratio only appeared in the SU(5) case.

We note that there are certain possible combinations of external fields which are not contained in the tables. There are four possible reasons for this. The first reason is that we have put into the Appendix again the cases where the messenger fields are Higgs-like and are thus not giving any new results beyond the renormalizable dimension 4 operators. The second reason is that, as in $\mathrm{SU}(5)$, the fields $X$ and $\bar{X}$ are not conjugated to each other introducing an extra model building complication which we do not want to discuss here. The third reason also appeared already in SU (5), namely the case when the product of the messengers contains more than one adjoint representation. In PS this happens for the 15-, 20- and 36-dimensional representation of $\mathrm{SU}(4)_{C}$. We have again dropped the nonunique

TABLE III. Summary of possible PS predictions for the GUT-scale Yukawa coupling ratios $\left(\left(Y_{e}\right)_{i j} /\left(Y_{d}\right)_{i j},\left(Y_{u}\right)_{i j} /\right.$ $\left.\left(Y_{d}\right)_{i j},\left(Y_{\nu}\right)_{i j} /\left(Y_{u}\right)_{i j}\right)$. The new relations compared to [9] are shown in bold. For more details about which operator gives which ratio, see Tables IV and V. Partial results for dimension 6 operators can be found in [10] where $\Lambda$ was also taken to be a singlet.

\begin{tabular}{lc}
\hline \hline Operator dimension $\left(\left(Y_{e}\right)_{i j} /\left(Y_{d}\right)_{i j},\left(Y_{u}\right)_{i j} /\left(Y_{d}\right)_{i j},\left(Y_{\nu}\right)_{i j} /\left(Y_{u}\right)_{i j}\right)$ \\
\hline 4 & $(1,1)$ \\
& $(-3,1)$ \\
& $(\mathbf{0}, \pm \mathbf{1})$ \\
& $(-\mathbf{1} / \mathbf{3}, \pm \mathbf{1})$ \\
$(1, \pm 1)$ \\
$(\mathbf{3} / \mathbf{2}, \pm \mathbf{1})$ \\
$(-3, \pm 1)$ \\
$(9, \pm 1)$ \\
\hline \hline
\end{tabular}


TABLE IV. Resulting predictions for the PS GUT-scale Yukawa coupling ratios $\left(\left(Y_{e}\right)_{i j} /\left(Y_{d}\right)_{i j},\left(Y_{u}\right)_{i j} /\left(Y_{d}\right)_{i j}\right.$, $\left.\left(Y_{\nu}\right)_{i j} /\left(Y_{u}\right)_{i j}\right)$ from the diagram in Fig. 1; for more details see main text.

\begin{tabular}{|c|c|c|c|c|}
\hline$\left(A, B_{1}\right)$ & $\left(C, B_{2}\right)$ & $X$ & $\Lambda$ & $\left(\left(Y_{e}\right)_{i j} /\left(Y_{d}\right)_{i j},\left(Y_{u}\right)_{i j} /\left(Y_{d}\right)_{i j},\left(Y_{\nu}\right)_{i j} /\left(Y_{u}\right)_{i j}\right)$ \\
\hline$\overline{\left(R, h_{1}\right)}$ & $\left(\bar{R}, \phi_{1}^{+}\right)$ & $(\overline{\mathbf{4}}, \mathbf{1}, \overline{\mathbf{2}})$ & $\Lambda_{1}^{ \pm}$ & $(1, \pm 1,1)$ \\
\hline$\left(R, h_{1}\right)$ & $\left(\bar{R}, \phi_{1}^{+}\right)$ & $(\overline{\mathbf{4}}, \mathbf{1}, \overline{2})$ & $\Lambda_{15}^{ \pm}$ & $(-1 / 3, \pm 1,-1 / 3)$ \\
\hline$\left(R, h_{1}\right)$ & $\left(\bar{R}, \phi_{1}^{-}\right)$ & $(\overline{\mathbf{4}}, \mathbf{1}, \overline{2})$ & $\Lambda_{1}^{ \pm}$ & $(1, \mp 1,1)$ \\
\hline$\left(R, h_{1}\right)$ & $\left(\bar{R}, \phi_{1}^{-}\right)$ & $(\overline{4}, 1, \overline{2})$ & $\Lambda_{15}^{ \pm}$ & $(-1 / 3, \mp 1,-1 / 3)$ \\
\hline$\left(R, h_{1}\right)$ & $\left(\bar{R}, \phi_{15}^{+}\right)$ & $(\overline{\mathbf{4}}, \mathbf{1}, \overline{2})$ & $\Lambda_{1}^{ \pm}$ & $(-3, \pm 1,-3)$ \\
\hline$\left(R, h_{1}\right)$ & $\left(\bar{R}, \phi_{15}^{+}\right)$ & $(\overline{\mathbf{4}}, \mathbf{1}, \overline{2})$ & $\Lambda_{15}^{ \pm}$ & $(1, \pm 1,1)$ \\
\hline$\left(R, h_{1}\right)$ & $\left(\bar{R}, \phi_{15}^{-}\right)$ & $(\overline{4}, 1, \overline{2})$ & $\Lambda_{1}^{ \pm}$ & $(-3, \mp 1,-3)$ \\
\hline$\left(R, h_{1}\right)$ & $\left(\bar{R}, \phi_{15}^{-}\right)$ & $(\overline{\mathbf{4}}, \mathbf{1}, \overline{2})$ & $\Lambda_{15}^{ \pm}$ & $(1, \mp 1,1)$ \\
\hline$\left(R, h_{15}\right)$ & $\left(\bar{R}, \phi_{1}^{+}\right)$ & $(\overline{\mathbf{4}}, \mathbf{1}, \overline{2})$ & $\Lambda_{1}^{ \pm}$ & $(-3, \pm 1,-3)$ \\
\hline$\left(R, h_{15}\right)$ & $\left(\bar{R}, \phi_{1}^{+}\right)$ & $(\overline{\mathbf{4}}, \mathbf{1}, \overline{\mathbf{2}})$ & $\Lambda_{15}^{ \pm}$ & $(1, \pm 1,1)$ \\
\hline$\left(R, h_{15}\right)$ & $\left(\bar{R}, \phi_{1}^{-}\right)$ & $(\overline{\mathbf{4}}, \mathbf{1}, \overline{2})$ & $\Lambda_{1}^{ \pm}$ & $(-3, \mp 1,-3)$ \\
\hline$\left(R, h_{15}\right)$ & $\left(\bar{R}, \phi_{1}^{-}\right)$ & $(\overline{\mathbf{4}}, \mathbf{1}, \overline{2})$ & $\Lambda_{15}^{ \pm}$ & $(1, \mp 1,1)$ \\
\hline$\left(R, h_{15}\right)$ & $\left(\bar{R}, \phi_{15}^{+}\right)$ & $(\overline{\mathbf{4}}, \mathbf{1}, \overline{2})$ & $\Lambda_{1}^{ \pm}$ & $(9, \pm 1,9)$ \\
\hline$\left(R, h_{15}\right)$ & $\left(\bar{R}, \phi_{15}^{+}\right)$ & $(\overline{\mathbf{4}}, \mathbf{1}, \overline{\mathbf{2}})$ & $\Lambda_{15}^{ \pm}$ & $(-3, \pm 1,-3)$ \\
\hline$\left(R, h_{15}\right)$ & $\left(\bar{R}, \phi_{15}^{-}\right)$ & $(\overline{\mathbf{4}}, \mathbf{1}, \overline{2})$ & $\Lambda_{1}^{ \pm}$ & $(9, \mp 1,9)$ \\
\hline$\left(R, h_{15}\right)$ & $\left(\bar{R}, \phi_{15}^{-}\right)$ & $(\overline{4}, \mathbf{1}, \overline{2})$ & $\Lambda_{15}^{ \pm}$ & $(-3, \mp 1,-3)$ \\
\hline$\left(R, h_{15}\right)$ & $\left(\bar{R}, \phi_{15}^{+}\right)$ & $(\overline{\mathbf{2 0}}, \mathbf{1}, \overline{2})$ & $\Lambda_{1}^{ \pm}$ & $(0, \pm 1,0)$ \\
\hline$\left(R, h_{15}\right)$ & $\left(\bar{R}, \phi_{15}^{+}\right)$ & $(\overline{20}, 1, \overline{2})$ & $\Lambda_{15}^{ \pm}$ & $(0, \pm 1,0)$ \\
\hline$\left(R, h_{15}\right)$ & $\left(\bar{R}, \phi_{15}^{-}\right)$ & $(\overline{20}, 1, \overline{2})$ & $\Lambda_{1}^{ \pm}$ & $(0, \mp 1,0)$ \\
\hline$\left(R, h_{15}\right)$ & $\left(\bar{R}, \phi_{15}^{-}\right)$ & $(\overline{\mathbf{2 0}}, \mathbf{1}, \overline{\mathbf{2}})$ & $\Lambda_{15}^{ \pm}$ & $(0, \mp 1,0)$ \\
\hline$\left(R, h_{15}\right)$ & $\left(\bar{R}, \phi_{15}^{+}\right)$ & $(\overline{36}, 1, \overline{2})$ & $\Lambda_{1}^{ \pm}$ & $(3 / 2, \pm 1,3 / 2)$ \\
\hline$\left(R, h_{15}\right)$ & $\left(\bar{R}, \phi_{15}^{-}\right)$ & $(\overline{36}, 1, \overline{2})$ & $\Lambda_{1}^{ \pm}$ & $(3 / 2, \mp 1,3 / 2)$ \\
\hline
\end{tabular}

TABLE V. Resulting predictions for the PS GUT-scale Yukawa coupling ratios $\left(\left(Y_{e}\right)_{i j} /\left(Y_{d}\right)_{i j},\left(Y_{u}\right)_{i j} /\left(Y_{d}\right)_{i j}\right.$, $\left.\left(Y_{\nu}\right)_{i j} /\left(Y_{u}\right)_{i j}\right)$ from the diagram in Fig. 1; for more details see main text.

\begin{tabular}{|c|c|c|c|c|}
\hline$\left(A, B_{1}\right)$ & $\left(C, B_{2}\right)$ & $X$ & $\Lambda$ & $\left(\left(Y_{e}\right)_{i j} /\left(Y_{d}\right)_{i j},\left(Y_{u}\right)_{i j} /\left(Y_{d}\right)_{i j},\left(Y_{\nu}\right)_{i j} /\left(Y_{u}\right)_{i j}\right)$ \\
\hline$\left(R, \phi_{1}^{-}\right)$ & $\left(\bar{R}, h_{1}\right)$ & $(\overline{\mathbf{4}}, \overline{\mathbf{2}}, \mathbf{3})$ & $\Lambda_{1}^{+}$ & $(1,-1,1)$ \\
\hline$\left(R, \phi_{1}^{-}\right)$ & $\left(\bar{R}, h_{1}\right)$ & $(\overline{4}, \overline{2}, 3)$ & $\Lambda_{15}^{+}$ & $(-1 / 3,-1,-1 / 3)$ \\
\hline$\left(R, \phi_{1}^{-}\right)$ & $\left(\bar{R}, h_{15}\right)$ & $(\overline{\mathbf{4}}, \overline{\mathbf{2}}, \mathbf{3})$ & $\Lambda_{1}^{+}$ & $(-3,-1,-3)$ \\
\hline$\left(R, \phi_{1}^{-}\right)$ & $\left(\bar{R}, h_{15}\right)$ & $(\overline{4}, \overline{2}, \mathbf{3})$ & $\Lambda_{15}^{+}$ & $(1,-1,1)$ \\
\hline$\left(R, \phi_{15}^{-}\right)$ & $\left(\bar{R}, h_{1}\right)$ & $(\overline{\mathbf{4}}, \overline{\mathbf{2}}, \mathbf{3})$ & $\Lambda_{1}^{+}$ & $(-3,-1,-3)$ \\
\hline$\left(R, \phi_{15}^{-}\right)$ & $\left(\bar{R}, h_{1}\right)$ & $(\overline{4}, \overline{2}, 3)$ & $\Lambda_{15}^{+}$ & $(1,-1,1)$ \\
\hline$\left(R, \phi_{15}^{-}\right)$ & $\left(\bar{R}, h_{15}\right)$ & $(\overline{4}, \overline{2}, \mathbf{3})$ & $\Lambda_{1}^{+}$ & $(9,-1,9)$ \\
\hline$\left(R, \phi_{15}^{-}\right)$ & $\left(\bar{R}, h_{15}\right)$ & $(\overline{\mathbf{4}}, \overline{\mathbf{2}}, \mathbf{3})$ & $\Lambda_{15}^{+}$ & $(-3,-1,-3)$ \\
\hline$\left(R, \phi_{1}^{+}\right)$ & $\left(\bar{R}, h_{1}\right)$ & $(\overline{\mathbf{4}}, \overline{\mathbf{2}}, \mathbf{1})$ & $\Lambda_{1}^{+}$ & $(1,1,1)$ \\
\hline$\left(R, \phi_{1}^{+}\right)$ & $\left(\bar{R}, h_{1}\right)$ & $(\overline{4}, \overline{2}, \mathbf{1})$ & $\Lambda_{15}^{+}$ & $(-1 / 3,1,-1 / 3)$ \\
\hline$\left(R, \phi_{1}^{+}\right)$ & $\left(\bar{R}, h_{15}\right)$ & $(\overline{\mathbf{4}}, \overline{\mathbf{2}}, \mathbf{1})$ & $\Lambda_{1}^{+}$ & $(-3,1,-3)$ \\
\hline$\left(R, \phi_{1}^{+}\right)$ & $\left(\bar{R}, h_{15}\right)$ & $(\overline{\mathbf{4}}, \overline{\mathbf{2}}, \mathbf{1})$ & $\Lambda_{15}^{+}$ & $(1,1,1)$ \\
\hline$\left(R, \phi_{15}^{+}\right)$ & $\left(\bar{R}, h_{1}\right)$ & $(\overline{\mathbf{4}}, \overline{\mathbf{2}}, \mathbf{1})$ & $\Lambda_{1}^{+}$ & $(-3,1,-3)$ \\
\hline$\left(R, \phi_{15}^{+}\right)$ & $\left(\bar{R}, h_{1}\right)$ & $(\overline{\mathbf{4}}, \overline{\mathbf{2}}, \mathbf{1})$ & $\Lambda_{15}^{+}$ & $(1,1,1)$ \\
\hline$\left(R, \phi_{15}^{+}\right)$ & $\left(\bar{R}, h_{15}\right)$ & $(\overline{\mathbf{4}}, \overline{\mathbf{2}}, \mathbf{1})$ & $\Lambda_{1}^{+}$ & $(9,1,9)$ \\
\hline$\left(R, \phi_{15}^{+}\right)$ & $\left(\bar{R}, h_{15}\right)$ & $(\overline{\mathbf{4}}, \overline{\mathbf{2}}, \mathbf{1})$ & $\Lambda_{15}^{+}$ & $(-3,1,-3)$ \\
\hline$\left(R, \phi_{15}^{+}\right)$ & $\left(\bar{R}, h_{15}\right)$ & $(\overline{\mathbf{2 0}}, \overline{\mathbf{2}}, \mathbf{1})$ & $\Lambda_{1}^{+}$ & $(0,1,0)$ \\
\hline$\left(R, \phi_{15}^{+}\right)$ & $\left(\bar{R}, h_{15}\right)$ & $(\overline{\mathbf{2 0}}, \overline{\mathbf{2}}, \mathbf{1})$ & $\Lambda_{15}^{+}$ & $(0,1,0)$ \\
\hline$\left(R, \phi_{15}^{-}\right)$ & $\left(\bar{R}, h_{15}\right)$ & $(\overline{\mathbf{2 0}}, \overline{2}, 3)$ & $\Lambda_{1}^{+}$ & $(0,-1,0)$ \\
\hline$\left(R, \phi_{15}^{-}\right)$ & $\left(\bar{R}, h_{15}\right)$ & $(\overline{\mathbf{2 0}}, \overline{\mathbf{2}}, \mathbf{3})$ & $\Lambda_{15}^{+}$ & $(0,-1,0)$ \\
\hline$\left(R, \phi_{15}^{+}\right)$ & $\left(\bar{R}, h_{15}\right)$ & $(\overline{\mathbf{3 6}}, \overline{\mathbf{2}}, \mathbf{1})$ & $\Lambda_{1}^{+}$ & $(3 / 2,1,3 / 2)$ \\
\hline$\left(R, \phi_{15}^{-}\right)$ & $\left(\bar{R}, h_{15}\right)$ & $(\overline{\mathbf{3 6}}, \overline{2}, 3)$ & $\Lambda_{1}^{+}$ & $(3 / 2,-1,3 / 2)$ \\
\hline
\end{tabular}


predictions which affected here only the 36-dimensional representations.

Apart from these three reasons, in the case of PS models another reason has emerged from our analysis, namely that it can happen that even if $X$ and $\bar{X}$ are in conjugated representations to each other, some messenger components remain massless if $\Lambda$ is a non-singlet. For the $\mathrm{SU}(2)_{R}$ part this can be understood easily as follows: Remembering that the adjoint is a traceless tensor we have

$$
\left\langle\Lambda_{1}^{-}\right\rangle=\Lambda\left(\begin{array}{cc}
1 & 0 \\
0 & -1
\end{array}\right) .
$$

Suppose now $X$ and $\bar{X}$ are adjoints of $\mathrm{SU}(2)_{R}$ as well with

$$
X=\left(\begin{array}{cc}
X^{0} & X^{+} \\
X^{-} & -X^{0}
\end{array}\right)
$$

and similar for $\bar{X}$. The mass term generated by the VEV of $\Lambda_{1}^{-}$is then

$$
\mathcal{W} \supset \operatorname{Tr}\left(X\left\langle\Lambda_{1}^{-}\right\rangle \bar{X}\right)=\Lambda\left(X^{+} \bar{X}^{-}+X^{-} \bar{X}^{+}\right),
$$

which implies that there is no mass term for $X^{0}$ and $\bar{X}^{0}$. Similar to the case where $X$ and $\bar{X}$ are not conjugated to each other this deficit might be fixed by introducing an extra field (in this case another $\Lambda$ field) which might however spoil the clean prediction so that we decided not to list such cases as well.

\section{GENERALIZATION TO HIGHER ORDER OPERATORS}

So far we have discussed only relations coming from diagrams with only one messenger pair $X$ and $\bar{X}$. Nevertheless, these results can be generalized to diagrams with additional external heavy fields and additional insertion of (nontrivial) messenger masses. Indeed, in such a way it is possible to realize new CG factors, which can be understood as products of the CG factors appearing at lower orders. There are basically three effects which have an influence on the Yukawa coupling ratio predicted by the respective diagram, as expounded in the following bullet points.

(i) Already at the renormalizable level the Yukawa coupling ratio depends on which representations contain the electroweak symmetry breaking Higgs doublets. In SU(5) these are $\bar{h}_{5}$ and $\bar{h}_{45}$ [and $h_{5}$ and $h_{45}$ which do not matter here because the up-type quark Yukawa couplings are not related to any other Yukawa couplings in SU(5)]. And in PS these are the fields $h_{1}$ and $h_{15}$. While $\bar{h}_{5}$ and $h_{1}$ give unification of Yukawa couplings, $\bar{h}_{45}$ and $h_{15}$ give a relative factor of -3 between leptons and quarks (the VEV points in the direction of $B-L$ ).

(ii) The second effect is associated with external fields receiving a GUT-scale VEV. Let us consider for example the $\mathrm{SU}(5)$ diagram from Fig. 1 with $\left(A, B_{1}\right)=\left(F, H_{24}\right) \quad$ with $\left(C, B_{2}\right)=\left(T, \bar{h}_{5}\right)$ and $X=\mathbf{5}$. Then $\left(Y_{e}\right)_{i j} /\left(Y_{d}\right)_{i j}=-3 / 2$ which is nothing else than the ratio of hypercharges of the fields contained in the $\mathbf{5}$ of SU(5) because the VEV of $H_{24}$ points into the hypercharge direction. Looking at Fig. 2 it is straightforward to see how this result generalizes: inserting an additional $H_{24}$ (as a $B_{j}$ in Fig. 2) coupling to five-plet messenger fields contributes a factor of $-3 / 2$ to the resulting $C G$ factor. The analogous consideration can be done for an additional $\mathrm{H}_{24}$ coupling to ten-plet messenger fields. This yields an additional factor of 6 . For the PS case similar arguments apply.

(iii) Thirdly, the resulting CG factor can be affected when the messenger masses arise from VEVs of $\Lambda_{i}$ fields which are not gauge singlets. This leads to split masses for the component fields of the messengers, and thus to inverse CG factors as we discussed in the previous sections. As shown in Fig. 2, also this mechanism can be generalized. In the $\mathrm{SU}(5)$ case, this generalization is particularly simple because the mass terms we consider are either universal or coming from an adjoint giving the inverse of the hypercharge ratio for the Yukawa coupling ratio. For each nontrivial messenger mass from an adjoint, we therefore obtain a factor of $(-3 / 2)^{-1}$ for five-dimensional messengers and a factor of $(1 / 6)^{-1}$ for ten-dimensional messengers. Again, similar considerations can be done for the PS case.

Based on these considerations, one can construct new diagrams which effectively generate products of CG factors. Instead of going through all possibilities at higher

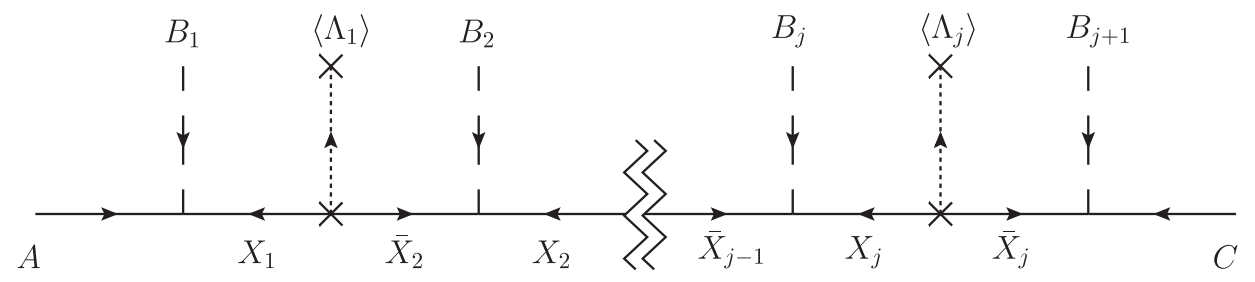

FIG. 2. Generalization of Fig. 1 with $j$ messenger pairs, $j$ insertions of $\Lambda$ VEVs and $j+1$ external $B$ fields from which one receives an electroweak VEV and $j$ receive high scale VEVs. 


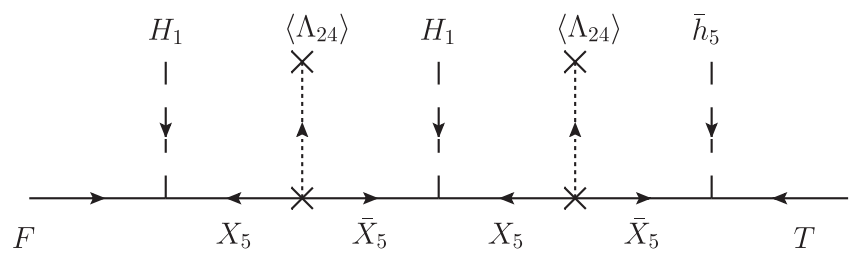

FIG. 3. Example for a generalization of Fig. 1 (of the form shown in Fig. 2) which leads to a CG factor of 4/9. The messenger pairs $X_{5}$ and $\bar{X}_{5}$ are five-dimensional representations of SU(5). The notation for the other fields and further details can be found in the main text. A possible application is discussed in Sec. V.

order, let us consider an explicit example (which we then also apply in the next section). Figure 3 shows a diagram which illustrates how a CG factor of $4 / 9$ can be realized. The two split messenger masses from the VEVs of adjoint representations each contribute a factor of $(-3 / 2)^{-1}$, such that the resulting CG factor is $(-3 / 2)^{-2}=4 / 9$.

\section{APPLICATIONS}

The novel CG factors found in the previous sections open up new possibilities for GUT model building. In this section, we will discuss some of these possibilities. In particular, the new CG factors allow for alternative textures for the GUT-scale Yukawa matrices, especially regarding the first two families. Let us briefly discuss the situation in $\mathrm{SU}(5)$ as an example (following $[18,19]$ ), where we have neglected the sign of the CG coefficients for the sake of simplicity.

In SU(5) GUTs, $Y_{e}$ is related to $Y_{d}^{T}$, and without loss of generality we can write for the upper 1-2 block describing the first two families in the $2 \times 2$ Yukawa matrices

$Y_{d}=\left(\begin{array}{ll}d & b \\ a & c\end{array}\right) \Rightarrow Y_{e}=\left(\begin{array}{cc}c_{d} d & c_{b} b \\ c_{a} a & c_{c} c\end{array}\right)^{T}=\left(\begin{array}{cc}c_{d} d & c_{a} a \\ c_{b} b & c_{c} c\end{array}\right)$

where we have introduced the CG factors $c_{a}, c_{b}, c_{c}$ and $c_{d}$ for the respective matrix elements. We assume here that each of the matrix elements arises dominantly from one GUT operator and that the 1-3 and 2-3 mixing effects in $Y_{d}$ and $Y_{e}$ can be neglected for discussing the mass relations for the first two families. Possible GUToperators and their predicted CG factors have been discussed in the previous sections. With $a, b, c$ and $d$ all being nonzero complex numbers, this obviously results in a large number of possibilities.

Many of these possibilities are however constrained by phenomenology. For instance in the context of SUSY GUTs, to check the validity of such a texture, one has to compute the renormalization group evolution of the mass eigenvalues from the GUT scale to low energies (or vice versa), taking into account the radiative threshold effects at the SUSY scale. In a recent study [20], the following constraints for the GUT-scale values of the diagonal Yukawa couplings have been derived:

$$
\frac{\left(1+\bar{\eta}_{\ell}\right) y_{\mu}}{\left(1+\bar{\eta}_{q}\right) y_{s}} \approx 4.36 \pm 0.23, \quad \frac{\left(1+\bar{\eta}_{\ell}\right) y_{e}}{\left(1+\bar{\eta}_{q}\right) y_{d}} \approx 0.41_{-0.06}^{+0.02},
$$

where the $\bar{\eta}_{i}$ are threshold correction parameters and where the ranges indicate the $1 \sigma$ uncertainties. The $\bar{\eta}_{i}$ enter both relations and they drop out in the following constraint equation for the effective CG factors $c_{e}=y_{e} / y_{d}$ and $c_{\mu}=y_{\mu} / y_{s}$, where $y_{e}, y_{\mu}, y_{d}$ and $y_{s}$ are the Yukawa couplings of the electron, the muon, the down quark and the strange quark, respectively [20]:

$$
\frac{y_{\mu}}{y_{s}}\left(\frac{y_{e}}{y_{d}}\right)^{-1}=\frac{c_{\mu}}{c_{e}} \approx 10.7_{-0.8}^{+1.8} .
$$

From this constraint, one can easily check the validity of the possible GUT textures for the masses of the first two families.

Another interesting consequence of these GUT textures is that, when embedded into a full flavor GUT model, they give different 1-2 mixing contributions in the charged lepton sector, as discussed systematically for the CG factors of $[9]$ in $[18,19]$. In small mixing approximation, it is given as $\theta_{12}^{\mathrm{e}} \approx \frac{c_{a} a}{c_{c} c}$. Its value (and phase) is important for selecting possibly viable textures of the neutrino mass matrix and for calculating the predictions for the leptonic mixing angles. For example, in combination with a neutrino mass matrix with zero 1-3 mixing and negligible 1-3 mixing in $Y_{e}$, the size of $\theta_{12}^{\mathrm{e}}$ controls the leptonic 1-3 mixing angle as $\theta_{13}^{\mathrm{PMNS}} \approx \sin \left(\theta_{23}^{\mathrm{PMNS}}\right) \theta_{12}^{\mathrm{e}} \approx \theta_{12}^{\mathrm{e}} / \sqrt{2}$. For possible choices of CG factors, which relate $\theta_{12}^{\mathrm{e}}$ to the Cabibbo angle $\theta_{C}$, the resulting predictions for $\theta_{13}^{\text {PMNS }}$ have been studied in $[18,19]$. The specific relation $\theta_{13}^{\mathrm{PMNS}} \approx \theta_{C} / \sqrt{2}$, which is close to the measured value, has been discussed recently in the context of GUTs in $[21,22]$.

\section{A. Example 1: Alternative textures with zero $\left(Y_{e}\right)_{11}$ and $\left(Y_{d}\right)_{11}$}

With $a, b, c$, and $d$ all nonzero complex numbers, there are in general no predictions for the quark-lepton mass ratios. Probably the most popular predictive texture in SU (5) GUT model building uses the Georgi-Jarlskog CG factor $c_{c}=3$ and $c_{a}=c_{b}=1$ while $d=0$, i.e.

$$
Y_{d}=\left(\begin{array}{cc}
0 & b \\
a & c
\end{array}\right) \Rightarrow Y_{e}=\left(\begin{array}{cc}
0 & b \\
a & 3 c
\end{array}\right)^{T}=\left(\begin{array}{cc}
0 & a \\
b & 3 c
\end{array}\right),
$$

often combined with the assumption of a symmetric matrix. This texture results (in leading order small mixing approximation) in diagonal Yukawa couplings $y_{d} \approx a b / c, y_{s} \approx c$, $y_{e} \approx a b /(3 c), \quad y_{\mu} \approx 3 c$ and hence ratios of diagonal Yukawa couplings $c_{e}=\frac{y_{e}}{y_{d}}$ and $c_{\mu}=\frac{y_{\mu}}{y_{s}}$ given by 


$$
c_{\mu} \approx c_{c}=3, \quad c_{e} \approx \frac{c_{a} c_{b}}{c_{c}}=\frac{1}{3} \Rightarrow \frac{c_{\mu}}{c_{e}} \approx 9 .
$$

In addition, assuming $\left|\theta_{12}^{\mathrm{d}}\right| \approx \theta_{C}$ (which implies $a \approx b$ ), it leads to a small charged lepton mixing contribution of about $\theta_{12}^{\mathrm{e}} \approx \frac{c_{a} a}{c_{c} c} \approx \theta_{C} / 3$.

An alternative texture, with $\left|\theta_{12}^{\mathrm{e}}\right| \approx \theta_{C}$, and better agreement with the experimental data, was highlighted in [22]:

$$
Y_{d}=\left(\begin{array}{cc}
0 & b \\
a & c
\end{array}\right) \Rightarrow Y_{e}=\left(\begin{array}{cc}
0 & \frac{1}{2} b \\
6 a & 6 c
\end{array}\right)^{T}=\left(\begin{array}{cc}
0 & 6 a \\
\frac{1}{2} b & 6 c
\end{array}\right)
$$

which implies diagonal Yukawa couplings $y_{d} \approx a b / c$, $y_{s} \approx c, y_{e} \approx a b /(2 c), y_{\mu} \approx 6 c$ and hence ratios of diagonal Yukawa couplings $c_{e}=\frac{y_{e}}{y_{d}}$ and $c_{\mu}=\frac{y_{\mu}}{y_{s}}$ given by

$$
c_{\mu} \approx c_{c}=6, \quad c_{e} \approx \frac{c_{a} c_{b}}{c_{c}}=\frac{1}{2} \Rightarrow \frac{c_{\mu}}{c_{e}} \approx 12 .
$$

In this texture $c_{a}=c_{c}$ and thus (taking $\left|\theta_{12}^{\mathrm{d}}\right| \approx \theta_{C}$ which implies $a \approx b$ ) one obtains $\left|\theta_{12}^{\mathrm{e}}\right| \approx \theta_{C}$. The texture has been applied recently to construct predictive flavor GUT models in $[23,24]$. In Table II we have presented a new way to obtain the CG factor $\frac{1}{2}$ using an "inverse CG factor."

With the new possible CG factor of $\frac{4}{9}$ from a higherdimensional operator as discussed in Sec. IV there is another potentially interesting option which is in good agreement with the data, namely

$$
Y_{d}=\left(\begin{array}{cc}
0 & b \\
a & c
\end{array}\right) \Rightarrow Y_{e}=\left(\begin{array}{cc}
0 & \frac{4}{9} b \\
\frac{9}{2} a & \frac{9}{2} c
\end{array}\right)^{T}=\left(\begin{array}{cc}
0 & \frac{9}{2} a \\
\frac{4}{9} b & \frac{9}{2} c
\end{array}\right) .
$$

It implies diagonal Yukawa couplings $y_{d} \approx a b / c, y_{s} \approx c$, $y_{e} \approx 4 a b /(9 c), y_{\mu} \approx 9 c / 2$ and hence ratios of diagonal Yukawa couplings $c_{e}=\frac{y_{e}}{y_{d}}$ and $c_{\mu}=\frac{y_{\mu}}{y_{s}}$ given by

$$
c_{\mu} \approx c_{c}=\frac{9}{2}, \quad c_{e} \approx \frac{c_{a} c_{b}}{c_{c}}=\frac{4}{9} \Rightarrow \frac{c_{\mu}}{c_{e}} \approx 10 .
$$

\section{B. Example 2: Alternative textures with diagonal $Y_{e}$ and $Y_{d}$}

Another highly predictive situation is the case that $Y_{e}$ and $Y_{d}$ are both diagonal, i.e. $a=b=0$ in the above notation,

$$
\begin{aligned}
& Y_{d}=\left(\begin{array}{ll}
d & 0 \\
0 & c
\end{array}\right)=\left(\begin{array}{cc}
y_{d} & 0 \\
0 & y_{s}
\end{array}\right) \Rightarrow \\
& Y_{e}=\left(\begin{array}{cc}
c_{d} d & 0 \\
0 & c_{c} c
\end{array}\right)=\left(\begin{array}{cc}
y_{e} & 0 \\
0 & y_{\mu}
\end{array}\right) .
\end{aligned}
$$

Then, the ratios of diagonal Yukawa couplings $c_{e}=\frac{y_{e}}{y_{d}}$ and $c_{\mu}=\frac{y_{\mu}}{y_{s}}$ are simply given by

$$
c_{e}=c_{d}, \quad c_{\mu}=c_{c} \Rightarrow \frac{c_{\mu}}{c_{e}}=\frac{c_{c}}{c_{d}} .
$$

The new CG factor of $\frac{1}{3}$, available in Pati-Salam models, can be used in the combination $c_{c}=3$ and $c_{d}=\frac{1}{3}$ to obtain the same prediction for the mass relations as from the Georgi-Jarlskog texture, with (as above) $\frac{c_{\mu}}{c_{e}} \approx 9$. In contrast to the Georgi-Jarlskog texture it yields no charged lepton 1-2 mixing, $\theta_{12}^{\mathrm{d}} \approx 0$, which is interesting in the context of neutrino mass textures which generate all lepton mixing (including $\theta_{13}^{\mathrm{PMNS}}$ ) already in the neutrino sector, e.g. $[25,26]$.

A viable option for $\mathrm{SU}(5)$ is the combination of $\mathrm{CG}$ factors $c_{c}=6$ and $c_{d}=\frac{1}{2}$. It yields

$$
c_{\mu}=c_{c}=6, \quad c_{e}=c_{d}=\frac{1}{2} \Rightarrow \frac{c_{\mu}}{c_{e}}=12.0 .
$$

In Table II we have presented a new way to obtain the CG factor $\frac{1}{2}$ using an inverse CG factor.

The CG factor $\frac{4}{9}$ from a higher-dimensional operator (cf. Sec. IV) can also be used for the first family with diagonal $Y_{e}$ and $Y_{d}$, such that $c_{e}=c_{d}=\frac{4}{9}$ directly. In combination with $c_{c}=\frac{9}{2}$, we obtain

$$
c_{\mu}=c_{c}=\frac{9}{2}, \quad c_{e}=c_{d}=\frac{4}{9} \Rightarrow \frac{c_{\mu}}{c_{e}}=10.1,
$$

in good agreement with experiments.

\section{CONCLUSIONS}

We have proposed new GUT predictions for the ratios of quark and lepton Yukawa couplings arising from splitting the masses of the messenger fields for the GUT-scale Yukawa operators by CG factors from GUT symmetry breaking. The effect is that the CG factors enter inversely in the predicted quark-lepton mass relations. This allows new fractional CG factors for the ratios of charged lepton to down-type quark Yukawa couplings such as $\frac{1}{6},-\frac{2}{3}$ in SU(5) or $-\frac{1}{3}, \frac{3}{2}$ in Pati-Salam, leading to new possible GUT predictions.

We have systematically constructed the new predictions that can be realized this way in SU(5) GUTs and Pati-Salam unified theories. The new predictions all arise from the types of diagrams in Fig. 1 and their generalization to higher orders in Fig. 2, in other words, diagrams in which the messenger fields are matterlike and receive their masses from non-singlet GUT representations getting VEVs. The resulting possible new predictions are indicated in bold in Tables I and III.

Note that the diagrams of the type shown in Fig. 4, where the messengers are Higgs-like fields, always lead to 


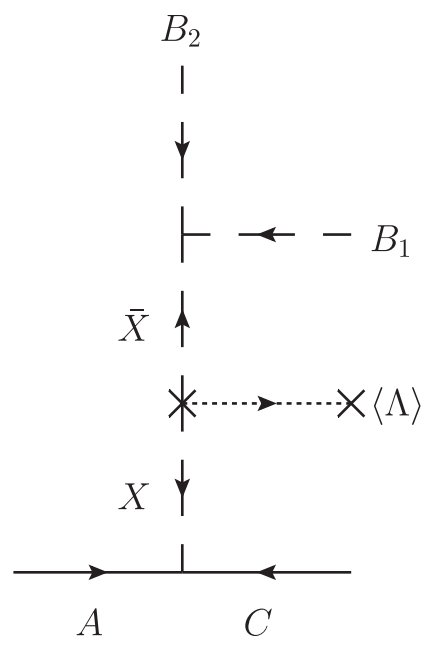

FIG. 4. Variation of Fig. 1 with a different topology where the messenger fields are "Higgs-like."

the usual CG relations, even when the messenger fields receive masses from non-singlet GUT representations getting VEVs. This is because in these types of diagrams, one may think of all such diagrams as giving an effective VEV for the Higgs coupling directly to the quarks and leptons.

We have also discussed some possible new model building applications involving the new CG coefficients in the case of both SU(5) GUTs and Pati-Salam unified theories. For example the new fractional CG coefficient of $\frac{4}{9}$ opens up some new interesting possibilities for $\mathrm{SU}(5)$ and the coefficient of $-\frac{1}{3}$ for Pati-Salam unified theories.

\section{ACKNOWLEDGMENTS}

We thank Vinzenz Maurer for useful discussions. S. A. acknowledges support by the Swiss National Science Foundation, S.F.K. from the STFC Consolidated ST/ J000396/1 and M.S. partially by the ERC Advanced Grant No. 267985 "DaMESyFla." S. F. K. and M. S. also acknowledge partial support from the EU Marie Curie ITN "UNILHC" (PITN-GA-2009-237920) and all authors were partially supported by the European Union under FP7 ITN INVISIBLES (Marie Curie Actions, PITN-GA-2011289442).

\section{CASES WITH HIGGS-LIKE MESSENGERS}

As discussed already in the main text we have put our main focus in this publication on diagrams like in Fig. 1 where $A$ and $C$ are GUT matter representations. For these diagrams the messengers are matterlike [they would carry a $\mathrm{U}(1)_{R}$ charge if we would introduce an $R$ symmetry]. In fact, only this class of diagrams generates new relations beyond the renormalizable ones.
TABLE VI. Resulting predictions for the SU(5) GUT-scale Yukawa coupling ratios $\left(Y_{e}\right)_{i j} /\left(Y_{d}\right)_{i j}$ from the diagram in Fig. 4; for more details see main text. If the messenger representation $X$ has an index, there is more than one way to combine the fields $A$ and $C$ or $B_{1}$ and $B_{2}$ to form this representation.

\begin{tabular}{|c|c|c|c|c|}
\hline$(A, C)$ & $\left(B_{1}, B_{2}\right)$ & $X$ & $\Lambda$ & $\left(Y_{e}\right)_{i j} /\left(Y_{d}\right)_{i j}$ \\
\hline$(F, T)$ & $\left(\bar{h}_{5}, H_{24}\right)$ & $\overline{5}$ & $\Lambda_{1}$ & 1 \\
\hline$(F, T)$ & $\left(\bar{h}_{5}, H_{24}\right)$ & $\overline{45}$ & $\Lambda_{1}$ & -3 \\
\hline$(F, T)$ & $\left(\bar{h}_{5}, H_{75}\right)$ & $\overline{45}$ & $\Lambda_{1}$ & -3 \\
\hline$(F, T)$ & $\left(\bar{h}_{45}, H_{24}\right)$ & $\overline{5}$ & $\Lambda_{1}$ & 1 \\
\hline$(F, T)$ & $\left(\bar{h}_{45}, H_{24}\right)$ & $\overline{\mathbf{4 5}}_{1}$ & $\Lambda_{1}$ & -3 \\
\hline$(F, T)$ & $\left(\bar{h}_{45}, H_{24}\right)$ & $\overline{\mathbf{4 5}}_{2}$ & $\Lambda_{1}$ & -3 \\
\hline$(F, T)$ & $\left(\bar{h}_{45}, H_{75}\right)$ & $\overline{5}$ & $\Lambda_{1}$ & 1 \\
\hline$(F, T)$ & $\left(\bar{h}_{45}, H_{75}\right)$ & $\overline{\mathbf{4 5}}_{1}$ & $\Lambda_{1}$ & -3 \\
\hline$(F, T)$ & $\left(\bar{h}_{45}, H_{75}\right)$ & $\overline{\mathbf{4 5}}_{2}$ & $\Lambda_{1}$ & -3 \\
\hline$(F, T)$ & $\left(\bar{h}_{5}, H_{1}\right)$ & $\overline{5}$ & $\Lambda_{24}$ & 1 \\
\hline$(F, T)$ & $\left(\bar{h}_{45}, H_{1}\right)$ & $\overline{45}$ & $\Lambda_{24}$ & -3 \\
\hline$(F, T)$ & $\left(\bar{h}_{5}, H_{24}\right)$ & $\overline{5}$ & $\Lambda_{24}$ & 1 \\
\hline$(F, T)$ & $\left(\bar{h}_{5}, H_{24}\right)$ & $\overline{45}$ & $\Lambda_{24}$ & -3 \\
\hline$(F, T)$ & $\left(\bar{h}_{5}, H_{75}\right)$ & $\overline{45}$ & $\Lambda_{24}$ & -3 \\
\hline$(F, T)$ & $\left(\bar{h}_{45}, H_{24}\right)$ & $\overline{5}$ & $\Lambda_{24}$ & 1 \\
\hline$(F, T)$ & $\left(\bar{h}_{45}, H_{24}\right)$ & $\overline{\mathbf{4 5}}_{1}$ & $\Lambda_{24}$ & -3 \\
\hline$(F, T)$ & $\left(\bar{h}_{45}, H_{24}\right)$ & $\overline{\mathbf{4 5}}_{2}$ & $\Lambda_{24}$ & -3 \\
\hline$(F, T)$ & $\left(\bar{h}_{45}, H_{75}\right)$ & $\overline{5}$ & $\Lambda_{24}$ & 1 \\
\hline$(F, T)$ & $\left(\bar{h}_{45}, H_{75}\right)$ & $\overline{\mathbf{4 5}}_{1}$ & $\Lambda_{24}$ & -3 \\
\hline$(F, T)$ & $\left(\bar{h}_{45}, H_{75}\right)$ & $\overline{\mathbf{4 5}}_{2}$ & $\Lambda_{24}$ & -3 \\
\hline
\end{tabular}

Nevertheless, for completeness we have collected here in the Appendix also the other cases. They are described by the diagram in Fig. 4. In this case the messengers are Higgs-like ( $X$ would carry no $R$ charge) and their

TABLE VII. Resulting predictions for the PS GUT-scale Yukawa coupling ratios $\left(\left(Y_{e}\right)_{i j} /\left(Y_{d}\right)_{i j},\left(Y_{u}\right)_{i j} /\left(Y_{d}\right)_{i j},\left(Y_{\nu}\right)_{i j} /\right.$ $\left.\left(Y_{u}\right)_{i j}\right)$ from the diagram in Fig. 4; for more details see main text. If the messenger representation $X$ has an index, there is more than one way to combine the fields $A$ and $C$ or $B_{1}$ and $B_{2}$ to form this representation.

\begin{tabular}{lcccc}
\hline \hline & & & $\left(\left(Y_{e}\right)_{i j} /\left(Y_{d}\right)_{i j},\left(Y_{u}\right)_{i j} /\left(Y_{d}\right)_{i j}\right.$ \\
$(A, C)$ & $\left(B_{1}, B_{2}\right)$ & $X$ & $\Lambda$ & $(1, \pm 1,1)$ \\
\hline$(R, \bar{R})$ & $\left(h_{1}, \phi_{1}^{+}\right)$ & $(\mathbf{1}, \overline{\mathbf{2}}, \mathbf{2})$ & $\Lambda_{1}^{ \pm}$ & $(1, \mp 1,1)$ \\
$(R, \bar{R})$ & $\left(h_{1}, \phi_{1}^{-}\right)$ & $(\mathbf{1}, \overline{\mathbf{2}}, \mathbf{2})$ & $\Lambda_{1}^{ \pm}$ & $(1, \pm 1,1)$ \\
$(R, \bar{R})$ & $\left(h_{15}, \phi_{15}^{+}\right)$ & $(\mathbf{1}, \overline{\mathbf{2}}, \mathbf{2})$ & $\Lambda_{1}^{ \pm}$ & $(1, \mp 1,1)$ \\
$(R, \bar{R})$ & $\left(h_{15}, \phi_{15}^{-}\right)$ & $(\mathbf{1}, \overline{\mathbf{2}}, \mathbf{2})$ & $\Lambda_{1}^{ \pm}$ & $(-3, \pm 1,-3)$ \\
$(R, \bar{R})$ & $\left(h_{15}, \phi_{1}^{+}\right)$ & $(\mathbf{1 5}, \overline{\mathbf{2}}, \mathbf{2})$ & $\Lambda_{1}^{ \pm}$ & $(-3, \pm 1,-3)$ \\
$(R, \bar{R})$ & $\left(h_{1}, \phi_{15}^{+}\right)$ & $(\mathbf{1 5}, \overline{\mathbf{2}}, \mathbf{2})$ & $\Lambda_{1}^{ \pm}$ & $(-3, \mp 1,-3)$ \\
$(R, \bar{R})$ & $\left(h_{1}, \phi_{15}^{-}\right)$ & $(\mathbf{1 5}, \overline{\mathbf{2}}, \mathbf{2})$ & $\Lambda_{1}^{ \pm}$ & $(-3, \mp 1,-3)$ \\
$(R, \bar{R})$ & $\left(h_{15}, \phi_{1}^{-}\right)$ & $(\mathbf{1 5}, \overline{\mathbf{2}}, \mathbf{2})$ & $\Lambda_{1}^{ \pm}$ & $(-3, \pm 1,-3)$ \\
$(R, \bar{R})$ & $\left(h_{15}, \phi_{15}^{+}\right)$ & $\left(\mathbf{1 5}_{1}, \overline{\mathbf{2}}, \mathbf{2}\right)$ & $\Lambda_{1}^{ \pm}$ & $(-3, \pm 1,-3)$ \\
$(R, \bar{R})$ & $\left(h_{15}, \phi_{15}^{+}\right)$ & $\left(\mathbf{1 5}_{2}, \overline{\mathbf{2}}, \mathbf{2}\right)$ & $\Lambda_{1}^{ \pm}$ & $(-3, \mp 1,-3)$ \\
$(R, \bar{R})$ & $\left(h_{15}, \phi_{15}^{-}\right)$ & $\left(\mathbf{1 5}_{1}, \overline{\mathbf{2}}, \mathbf{2}\right)$ & $\Lambda_{1}^{ \pm}$ & $(-3, \mp 1,-3)$ \\
$(R, \bar{R})$ & $\left(h_{15}, \phi_{15}^{-}\right)$ & $\left(\mathbf{1 5}_{2}, \overline{\mathbf{2}}, \mathbf{2}\right)$ & $\Lambda_{1}^{ \pm}$ & \\
\hline \hline
\end{tabular}


representation determines the Yukawa coupling ratio. But since the product of the matter fields $A$ and $C$ allows only two possible representations containing a Higgs doublet we end up again with the two renormalizable Yukawa coupling ratios. For completeness we have collected the results for SU(5) in Table VI and for PS in Table VII.

Note again that we do not consider cases here where components of the messenger pairs $X$ and $\bar{X}$ remain massless.
[1] H. Georgi and S. L. Glashow, Phys. Rev. Lett. 32, 438 (1974).

[2] J. C. Pati and A. Salam, Phys. Rev. D 10, 275 (1974); 11, 703(E) (1975).

[3] H. Georgi and C. Jarlskog, Phys. Lett. 86B, 297 (1979).

[4] G. Ross and M. Serna, Phys. Lett. B 664, 97 (2008).

[5] W. Altmannshofer, D. Guadagnoli, S. Raby, and D. M. Straub, Phys. Lett. B 668, 385 (2008).

[6] H. Baer, S. Kraml, A. Lessa, and S. Sekmen, J. High Energy Phys. 02 (2010) 055.

[7] I. Gogoladze, R. Khalid, and Q. Shafi, Phys. Rev. D 79, 115004 (2009); I. Gogoladze, S. Raza, and Q. Shafi, Phys. Lett. B 706, 345 (2012); S. Dar, I. Gogoladze, Q. Shafi, and C. S. Un, Phys. Rev. D 84, 085015 (2011); I. Gogoladze, Q. Shaf,i, and C. S. Un, J. High Energy Phys. 08 (2012) 028; H. Baer, I. Gogoladze, A. Mustafayev, S. Raza, and Q. Shafi, J. High Energy Phys. 03 (2012) 047; I. Gogoladze, Q. Shafi, and C. S. Un, J. High Energy Phys. 07 (2012) 055; M. Adeel Ajaib, I. Gogoladze, Q. Shafi, and C. S. Un, J. High Energy Phys. 07 (2013) 139; M. A. Ajaib, I. Gogoladze, Q. Shafi, and C. S. Un, arXiv:1308.4652.

[8] A. Anandakrishnan, S. Raby, and A. Wingerter, Phys. Rev. D 87, 055005 (2013); A. Anandakrishnan, B. C. Bryant, S. Raby, and A. Wingerter, Phys. Rev. D 88, 075002 (2013).

[9] S. Antusch and M. Spinrath, Phys. Rev. D 79, 095004 (2009).

[10] B. C. Allanach, S. F. King, G. K. Leontaris, and S. Lola, Phys. Rev. D 56, 2632 (1997).
[11] B. C. Allanach, S. F. King, G. K. Leontaris, and S. Lola, Phys. Lett. B 407, 275 (1997).

[12] G. Anderson, S. Raby, S. Dimopoulos, L. J. Hall, and G. D. Starkman, Phys. Rev. D 49, 3660 (1994).

[13] F. Bazzocchi, M. Frigerio, and S. Morisi, Phys. Rev. D 78, 116018 (2008).

[14] M. Monaco and M. Spinrath, Phys. Rev. D 84, 055009 (2011).

[15] S. Antusch, L. Calibbi, V. Maurer, and M. Spinrath, Nucl. Phys. B852, 108 (2011).

[16] S. Antusch, L. Calibbi, V. Maurer, M. Monaco, and M. Spinrath, Phys. Rev. D 85, 035025 (2012).

[17] C. S. Aulakh and S. K. Garg, Nucl. Phys. B857, 101 (2012).

[18] S. Antusch and V. Maurer, Phys. Rev. D 84, 117301 (2011).

[19] D. Marzocca, S. T. Petcov, A. Romanino, and M. Spinrath, J. High Energy Phys. 11 (2011) 009.

[20] S. Antusch and V. Maurer, J. High Energy Phys. 11 (2013) 115.

[21] S. F. King, Phys. Lett. B 718, 136 (2012).

[22] S. Antusch, C. Gross, V. Maurer, and C. Sluka, Nucl. Phys. B866, 255 (2013).

[23] S. Antusch, C. Gross, V. Maurer, and C. Sluka, Nucl. Phys. B877, 772 (2013).

[24] S. Antusch, C. Gross, V. Maurer, and C. Sluka, Nucl. Phys. B879, 19 (2014).

[25] S. F. King, J. High Energy Phys. 07 (2013) 137.

[26] S. F. King, Phys. Lett. B 724, 92 (2013). 